\title{
Asymptotic behavior and moderate deviation principle for the maximum of a Dyck path
}

\author{
Termeh Kousha \\ School of Mathematics and Statistics, University of Ottawa \\ 585 King Edward Ottawa, ON K1N 6N5 \\ e-mail: tkous045@uottawa.ca
}

\begin{abstract}
In this paper, we obtain a large and moderate deviation principle for the law of the maximum of a random Dyck path. Our result extends the results of Chung [1], Kennedy [6] and Khorunzhiy and Marckert [7.

Keywords: Dyck paths, Catalan number, Moderate deviation principle, Brownian excursion.
\end{abstract}

\section{Introduction.}

Let $W(t), 0 \leq t<\infty$ denote a standard Brownian motion and define $\tau_{1}=\sup \{t<1: W(t)=0\}$ and $\tau_{2}=\inf \{t>1: W(t)=0\}$. Define the process $W_{1}(s), 0 \leq s \leq 1$ by setting

$$
W_{1}(s)=\frac{\left|W\left(s \tau_{2}+(1-s) \tau_{1}\right)\right|}{\left(\tau_{2}-\tau_{1}\right)^{\frac{1}{2}}} .
$$

The process $W_{1}$ is known as the unsigned, scaled Brownian excursion process [6]. Chung [1] and Kennedy [6] derived the distribution of the maximum of the unsigned scaled Brownian excursion.

For a path in the lattice $\mathbb{Z}^{2}$ the NE-SE path is a path which starts at $(0,0)$ and makes steps either of the form $(1,1)$ (North-East steps) or of the form $(1,-1)$ (South-East steps). A Dyck path is a NE-SE path which ends on the $x$-axis 
and never goes below the $x$-axis. It is known that after normalization a Dyck path converges to $W_{1}$ in distribution [5]. For any integer $N>0$, define the set of Dyck paths with length of $2 N$ as follows,

$D_{2 N}=\left\{S:=\left(S_{i}\right)_{0 \leq i \leq 2 N}: S_{0}=S_{2 N}=0, S_{i+1}=S_{i} \pm 1, S_{i} \geq 0 \forall i \in[0,2 N-1]\right\}$.

Khorunzhiy and Marckert [7] showed that for any $\lambda>0$,

$$
E\left(\exp \left(\lambda\left(2 N^{-\frac{1}{2}} \max _{1 \leq i \leq 2 N} S_{i}\right)\right)\right)
$$

converges and coincides with the moment generating functions of the normalized Brownian excursion on $[0,1]$.

In this paper, by using other methods, relying on the spectral properties of an associated adjacency matrix, we find the distribution of the maximum of a Dyck path and show that it has the same distribution function as the unsigned Brownian excursion which was first derived in 1976 by Kennedy [6]. We also obtain large and moderate deviation principles for the law of the maximum of a random Dyck path.

The paper is organized as follows. In Section 2 we start by giving some notation and finding a representation of the Catalan number. Using this representation we find the distribution of the maximum of the Dyck path for the case where the length of the Dyck path is proportional to the square root of the height. This result is already known but we reprove it by a different approach. In Section 3, we consider different cases and we find moderate and large deviation principles for the law of the maximum of a random Dyck path for those cases.

\section{Notation and some results}

For any integer $N \geq 0$, we denote by $C_{N}$ the $N$ th Catalan number,

$$
C_{N}:=\frac{1}{N+1}\left(\begin{array}{c}
2 N \\
N
\end{array}\right)=\frac{(2 N) !}{(N) !(N+1) !} .
$$

It is well known that $C_{N}=\left|D_{2 N}\right|$ where $|x|$ gives the cardinality of $x$.

From Stirling's formula, as $N$ goes to infinity the Catalan number satisfies $C_{N}=\frac{4^{N}}{(N)^{\frac{3}{2}} \sqrt{\pi}}(1+o(1))$.

Let $D_{2 N, n}=\left\{S: S \in D_{2 N}, \max _{1 \leq i \leq 2 N} S_{i}<n\right\}$. Note that if $N<n$ then $D_{2 N, n}=D_{2 N}$. 
Lemma 2.1. For positive integers $n$ and $N$, we have

$$
\left|D_{2 N, n}\right|=\sum_{s=1}^{n}\left(\frac{2}{n+1}\right) \sin ^{2}\left(\frac{\pi s}{n+1}\right)\left(2 \cos \left(\frac{\pi s}{n+1}\right)\right)^{2 N} .
$$

See [4, p.329] for the proof. An alternative proof below is provided relying on the spectral properties of an associated adjacency matrix , cf also [8, p.25]. As a corollary, we obtain a formula for the Catalan number.

Sketch of the proof. We consider the graph with $n$ vertices $\{1, \ldots, n\}$ and $n-1$ edges $\{\{1,2\},\{2,3\}, \ldots\{n-1, n\}\}$. This is the graph of a tree (and actually, of a line). Let $T=\left(t_{i j}\right)_{n \times n}$ be the adjacency matrix of this graph, i.e., $t_{i j}=\delta_{i, j+1}+\delta_{i, j-1}$ where $\delta_{i, j}$ is the Kronecker delta. We observe that,

$$
\left|D_{2 N, n}\right|=\left(T^{2 N}\right)_{11} \text {. }
$$

This follows directly from the definition of $D_{2 N, n}$ and from properties of adjacency graphs. Alternatively, we can write

$$
\left|D_{2 N, n}\right|=v^{T} T^{2 N} v
$$

where $v=(1,0, \ldots, 0)^{T}$. According to J. L. Lagrange's computation in 1759 [8], we have

$$
T=U D U^{T}
$$

where

$$
D=\operatorname{diag}\left(2 \cos \left(\frac{\pi k}{n+1}\right)\right)_{k=1}^{n},
$$

and the entries of the unitary matrix $U$ satisfy

$$
U_{k l}=\sin \left(\frac{k l \pi}{n+1}\right) \sqrt{\frac{2}{n+1}} .
$$

Putting these facts together and performing the matrix multiplication yields the desired conclusion.

Corollary 2.1. For positive integers $n>0$ and $0<N \leq n-1$, the $N$ th Catalan number satisfies $C_{N}=\left|D_{2 N}\right|=\left|D_{2 N, n}\right|$. Therefore,

$$
C_{N}=\sum_{s=1}^{n}\left(\frac{2}{n+1}\right) \sin ^{2}\left(\frac{\pi s}{n+1}\right)\left(2 \cos \left(\frac{\pi s}{n+1}\right)\right)^{2 N} .
$$


If $\mathbb{P}_{N}$ is the uniform distribution on $D_{2 N}$, then

$$
\frac{\left|D_{2 N, n}\right|}{C_{N}}=\mathbb{P}_{N}(\max \text { height of the Dyck paths in } 2 N \text { steps }<n) .
$$

We recall the following result. It follows from elementary calculus, so we omit its proof.

Lemma 2.2. For all $0 \leq x \leq \frac{\pi}{2}$, we have

$$
\cos (x) \leq \exp \left(-\frac{x^{2}}{2}\right)
$$

For positive integers $n, N$ and $s$ define,

$$
G_{N, n}(s)=\sin ^{2}\left(\frac{\pi s}{n+1}\right) \cos ^{2 N}\left(\frac{\pi s}{n+1}\right) .
$$

For a fixed $n$ we have $\sin \left(\frac{\pi}{n+1}\right)=\sin \left(\frac{n \pi}{n+1}\right)$ and $\cos \left(\frac{\pi}{n+1}\right)=-\cos \left(\frac{n \pi}{n+1}\right)$. So by symmetry we have $G_{N, n}(1)=G_{N, n}(n)$ and for even $n, \sum_{s=1}^{n} G_{N, n}(s)=$ $2 \sum_{s=1}^{\frac{n}{2}} G_{N, n}(s)$. Note that, without lost of generality for large value of $n$, we can assume $n$ is even. Since if $n$ is odd we have the sum is equal to $2 \sum_{s=1}^{\frac{n-1}{2}} G_{N, n}(s)+\sin ^{2}\left(\frac{n \pi}{n+1}\right)\left(2 \cos \left(\frac{n \pi}{n+1}\right)\right)^{2 N}$ and as $n \rightarrow \infty$ the last term will go to zero.

Let $[x]$ be the largest integer less than or equal to $\mathrm{x}$. The following result can be obtained from Fibonacci-Chebyshev polynomials [4, p.329] and applying Lagrange-Bürmann inversion theorem [4, p.732]. Below we give an alternative proof in the spirit of this paper using a direct asymptotic analytic approach.

Theorem 2.1. Let $N=\left[\operatorname{tn}^{2}\right]$ where $t$ is any positive number. We have

$$
\lim _{n \rightarrow \infty} \frac{\left|D_{2 N, n}\right|}{C_{N}}=f(t)
$$

where $f(t)=4 \sqrt{\pi} t^{\frac{3}{2}} \sum_{s=1}^{\infty} s^{2} \pi^{2} \exp \left(-t s^{2} \pi^{2}\right)$.

Proof. We have by symmetry,

$$
\begin{aligned}
\frac{\left|D_{2 N, n}\right|}{C_{N}} & =\frac{2 \frac{4^{N}}{(n+1)^{3}} \sum_{s=1}^{n}(n+1)^{2} G_{N, n}(s)}{\frac{4^{N}}{\sqrt{\pi} N^{\frac{3}{2}}}} \\
& =\frac{\frac{4^{N+1}}{(n+1)^{3}} \sum_{s=1}^{\frac{n}{2}}(n+1)^{2} G_{N, n}(s)}{\frac{4^{N}}{\sqrt{\pi} N^{\frac{3}{2}}}}
\end{aligned}
$$


We split the sum into two cases where $1 \leq s \leq \frac{\sqrt{n+1}}{(\ln n)^{\frac{1}{4}}}$ and $\frac{\sqrt{n+1}}{(\ln n)^{\frac{1}{4}}} \leq s \leq \frac{n}{2}$. By Lemma 2.2, for all $1 \leq s \leq \frac{n}{2}$, we have

$$
\cos \left(\frac{\pi s}{n+1}\right) \leq \exp \left(-\frac{\pi^{2} s^{2}}{2(n+1)^{2}}\right)
$$

For all $\frac{\sqrt{n+1}}{(\ln n)^{\frac{1}{4}}} \leq s \leq \frac{n}{2}$, we have

$$
G_{t n^{2}, n}(s) \leq \exp \left(\frac{-\pi^{2}(n+1)}{\sqrt{\ln n}}\right)
$$

Since $\cos \left(\frac{\pi s}{n+1}\right)$ decreases on this interval. Thus

$$
\begin{aligned}
\lim _{n \rightarrow \infty}(n+1)^{2} \sum_{s=\frac{\sqrt{n+1}}{(\ln n)^{\frac{1}{4}}}}^{\frac{n}{2}} G_{t n^{2}, n}(s) & \leq \lim _{n \rightarrow \infty}(n+1)^{2} \frac{n}{2} \exp \left(\frac{-\pi^{2}(n+1)}{\sqrt{\ln n}}\right) \\
& \rightarrow 0 .
\end{aligned}
$$

Let $g(s)=\exp \left(-t \pi^{2} s^{2}\right)$, then $g$ is a real valued function and $\sum_{s=1}^{\infty} g(s)<\infty$. So we have

$$
\sum_{s=1}^{n} \cos \left(\frac{\pi s}{n+1}\right)^{2 t n^{2}} \leq \sum_{s=1}^{n} \exp \left(\frac{-t \pi^{2} s^{2} n^{2}}{(n+1)^{2}}\right)<\infty .
$$

Moreover, we have for all $1 \leq s \leq \frac{\sqrt{n+1}}{(\ln n)^{\frac{1}{4}}}$ fixed,

$$
\begin{aligned}
& \lim _{n \rightarrow \infty} \cos \left(\frac{\pi s}{n+1}\right)^{2 t n^{2}} \rightarrow g(s) \text { and, } \\
& \lim _{n \rightarrow \infty}(n+1)^{2} \sin ^{2}\left(\frac{\pi s}{n+1}\right) \rightarrow \pi^{2} s^{2} .
\end{aligned}
$$

Hence for all $1 \leq s \leq \frac{\sqrt{n+1}}{(\ln n)^{\frac{1}{4}}},(n+1)^{2} G_{t n^{2}, n}(s)$ converges pointwise to $s^{2} \pi^{2} g(s)$. We also have,

$$
n^{2} \sin ^{2}\left(\frac{\pi s}{n+1}\right) \leq n^{2} \frac{\pi^{2} s^{2}}{(n+1)^{2}}<\pi^{2} s^{2}
$$


So $n^{2} \sin ^{2}\left(\frac{\pi s}{n+1}\right) \cos \left(\frac{\pi s}{n+1}\right)^{2 t n^{2}}<s^{2} \pi^{2} g(s)$, where $\sum_{s=1}^{\infty} s^{2} \pi^{2} g(s)<\frac{1}{4 t^{\frac{3}{2}} \sqrt{\pi}}$. So by Dominated Convergence Theorem we have,

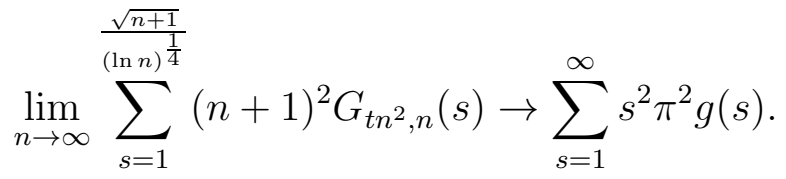

$$
\begin{aligned}
& \lim _{n \rightarrow \infty} \frac{\left|D_{2 t n^{2}, n}\right|}{C_{N}}=\lim _{n \rightarrow \infty} \frac{\frac{4^{t n^{2}+1}}{(n+1)^{3}} \sum_{s=1}^{\frac{\sqrt{n+1}}{(\ln n)^{\frac{1}{4}}}}(n+1)^{2} G_{t n^{2}, n}(s)}{\frac{4^{t n^{2}}}{\sqrt{\pi} t^{\frac{3}{2}} n^{3}}} \\
& +\lim _{n \rightarrow \infty} \frac{4 t^{\frac{3}{2}} \sqrt{\pi} n^{3}}{(n+1)^{3}} \sum_{\frac{\sqrt{n+1}}{(\ln n)^{\frac{1}{4}}}}^{\frac{n}{2}}(n+1)^{2} G_{t n^{2}, n}(s) \\
& =\lim _{n \rightarrow \infty} \frac{4\left[4^{t n^{2}}\left(\frac{1}{(n+1)^{3}}\right)\right]}{\frac{4^{t n^{2}}}{\sqrt{\pi} t^{\frac{3}{2}} n^{3}}} \sum_{s=1}^{\infty} s^{2} \pi^{2} \exp \left(-t s^{2} \pi^{2}\right)+0 \\
& =4 \sqrt{\pi} t^{\frac{3}{2}} \sum_{s=1}^{\infty} s^{2} \pi^{2} \exp \left(-t s^{2} \pi^{2}\right) \text {. }
\end{aligned}
$$

Let $x \sqrt{2 N}=n$, that is $x^{2}=\frac{1}{2 t}$ or equivalently $t=\frac{1}{2 x^{2}}$. We can reformulate our result as:

$$
f(x):=\lim _{N \rightarrow \infty} \mathbb{P}_{N}\left(\max _{0 \leq i \leq n} S_{i} \leq x \sqrt{2 N}\right)=4 \sqrt{\pi}(2)^{-\frac{3}{2}} x^{-3} \sum_{s=1}^{\infty} s^{2} \pi^{2} \exp \left(-\frac{\pi^{2} s^{2}}{2 x^{2}}\right) .
$$

Let $K$ denote the function used by Kennedy [6] and Chung [1] as the distribution of the maximum of the unsigned scaled Brownian excursion,

$K(x):=\mathbb{P}\left(\max _{s \in[0,1]} W_{1}(s) \leq x\right)=1-2 \sum_{s=1}^{\infty}\left(4 x^{2} s^{2}-1\right) \exp \left(-2 x^{2} s^{2}\right)$, for $x>0$.

Our Theorem [2.1 together with [6], [1], and [7] implies the following. Note that the following theorem is just a consequence of the Jacobis's functional equation. See [2, p.2] for an alternative proof arrived by Chung 1976 and [5, Theorem 2.6]. 
Theorem 2.2. For every $x>0, f(x)=K(x)$.

Proof. From Theorem 2.1, we have

$$
f(x)=\lim _{N \rightarrow \infty} \mathbb{P}_{N}\left(\max _{0 \leq i \leq n} S_{i} \leq x \sqrt{N}\right)=\sqrt{2 \pi} x^{-3} \sum_{s=1}^{\infty} s^{2} \pi^{2} \exp \left(-\frac{s^{2} \pi^{2}}{2 x^{2}}\right) .
$$

Alternatively, this result is proved below using the Poisson summation formula suggested by Dr. Rouault [9].

Alternative proof of Theorem 2.2. For an appropriate function $f$, the Poisson summation formula may be stated as:

$$
\sum_{n=-\infty}^{\infty} f(t+n T)=\frac{1}{T} \sum_{k=-\infty}^{\infty} \hat{f}\left(\frac{k}{T}\right) \exp \left(2 \pi i \frac{k}{T} t\right),
$$

where $\hat{f}$ is the Fourier transform of $f$. Let $f(x)=\exp \left(-2 x^{2}\right)$, so $\hat{f}=$ $\sqrt{\frac{\pi}{2}} \exp \left(-\frac{k^{2} \pi^{2}}{2}\right)$. Therefore, by Poisson summation formula with $t=0$ and $T=x$ we have,

$$
\sum_{n=-\infty}^{\infty} f(n x)=\frac{1}{x} \sum_{k=-\infty}^{\infty} \hat{f}\left(\frac{k}{x}\right)
$$

i.e.,

$$
\sum_{n=-\infty}^{\infty} \exp \left(-2 n^{2} x^{2}\right)=\frac{1}{x} \sum_{k=-\infty}^{\infty} \sqrt{\frac{\pi}{2}} \exp \left(\frac{-\pi^{2} k^{2}}{2 x^{2}}\right) .
$$

Separating positive and negative indices and multiplying by $x$ yields

$$
x\left(1+2 \sum_{n=1}^{\infty} \exp \left(-2 n^{2} x^{2}\right)\right)=1+2 \sum_{k=1}^{\infty} \sqrt{\frac{\pi}{2}} \exp \left(\frac{-\pi^{2} k^{2}}{2 x^{2}}\right) .
$$

Now by taking the derivative with respect to $x$ we get,

$$
1-2 \sum_{n=1}^{\infty} \exp \left(-2 n^{2} x^{2}\right)\left(4 n^{2} x^{2}-1\right)=\sqrt{2 \pi} \frac{\pi^{2} k^{2}}{x^{3}} \sum_{k=1}^{\infty} \exp \left(\frac{-\pi^{2} k^{2}}{2 x^{2}}\right),
$$

which implies $f(x)=K(x)$ and completes our proof. 


\section{$3 \quad$ Large and moderate deviation principle for the maximum of Dyck paths}

Large deviation theory deals with the decay of the probability of increasingly unlikely events. Historically, the oldest and most important result is that the empirical mean of an i.i.d. sequence of real-valued random variables obeys a large deviation principle with rate $n$.

Theorem 3.1 (Cramér's Theorem). Let $X_{1}, X_{2}, \cdots$ be i.i.d. random variables with mean $\mu$ satisfying $\varphi(\lambda):=\log \left(\mathbb{E} e^{\lambda X}\right)<\infty$, and let $S_{n}:=\sum_{i=1}^{n} X_{i}$. For any $x>\mu$ we have,

$$
\lim _{n \rightarrow \infty} \frac{1}{n} \log \mathbb{P}\left\{\frac{1}{n} S_{n} \geq x\right\}=-\varphi^{*}(x),
$$

where $\varphi^{*}(x)$, given by $\varphi^{*}(x):=\sup _{\lambda \in \mathbb{R}}\{\lambda x-\varphi(\lambda)\}$ is the Legendre transform of $\varphi$.

The moderate deviation principle gives us the probability of deviation of $S_{n}$ of size $a_{n}$ where $a_{n} \ll n$, i.e., $\frac{a_{n}}{n}=o(1)$.

Theorem 3.2 (Moderate deviation principle for a random walk). Under the same assumptions as in Theorem [3.1, if $\sqrt{n} \ll a_{n} \ll n$ we have, for all $x>0$,

$$
\lim _{n \rightarrow \infty} \frac{n}{a_{n}^{2}} \log \mathbb{P}\left\{S_{n}-\mu n \geq x a_{n}\right\}=-\frac{x^{2}}{2 \sigma^{2}} .
$$

In this section, we discuss the large and moderate deviation principle for the maximum of the Dyck path in two cases. We start with the following lemma which we will use later.

Lemma 3.1. For a fixed $n$ and $N \gg n^{2}$. We have for any $1<s<n$,

$$
\frac{G_{N, n}(s)}{G_{N, n}(n)}=o(1) \text { as } N \rightarrow \infty
$$

and even $G_{N, n}(1) \gg \sum_{s=2}^{n-1} G_{N, n}(s)$. Therefore,

$$
\sum_{s=1}^{n} G_{N, n}(s)=2 G_{N, n}(1)+o(1) .
$$


We omit the details of the proof, but it follows from Lemma 2.2 and by an application of the Dominated Convergence Theorem.

Remark 1. It also follows from the Dominated Convergence Theorem that,

$$
\lim _{n \rightarrow \infty} \frac{\left|D_{2 N, n}\right|}{C_{N}}= \begin{cases}0, & N \gg n^{2} ; \\ 1, & n \ll N \ll n^{2} .\end{cases}
$$

We obtain the following large and moderate deviation principle for a random Dyck path when $N \gg n^{2}$. Note that $n$ might be any function of $N$ as long as $N \gg n^{2}$.

Theorem 3.3. Let $N$ and $n$ be positive integers satisfying $N \gg n^{2}$. Then

$\lim _{N \rightarrow \infty} \frac{(n+1)^{2}}{N} \log \mathbb{P}_{N}($ max height of the Dyck Path with length $2 N<n) \longrightarrow-\pi^{2}$.

Proof. For sufficiently large $N$ we have,

$$
\begin{aligned}
\frac{\left|D_{2 N, n}\right|}{C_{N}} & =\frac{\sum_{s=1}^{n} \frac{2}{n+1} \sin ^{2}\left(\frac{\pi s}{n+1}\right) 4^{N} \cos ^{2 N}\left(\frac{\pi s}{n+1}\right)}{\frac{4^{N}}{\sqrt{\pi} N^{\frac{3}{2}}}} \\
& =\frac{2}{(n+1)} \sqrt{\pi} N^{\frac{3}{2}} \sum_{s=1}^{n} G_{N, n}(s),
\end{aligned}
$$

From Lemma 3.1, we know that for sufficiently large $N$ we have

$$
\begin{aligned}
\frac{2}{(n+1)} \sqrt{\pi} N^{\frac{3}{2}} \sum_{s=1}^{n} G_{N, n}(s) & =\frac{2}{(n+1)} \sqrt{\pi} N^{\frac{3}{2}}\left(2 G_{N, n}(1)+o(1)\right) \\
& =\frac{4 \sqrt{\pi} N^{\frac{3}{2}}}{n+1} \sin ^{2}\left(\frac{\pi}{n+1}\right) \cos ^{2 N}\left(\frac{\pi}{n+1}\right)(1+o(1)) \\
& =\frac{4 \sqrt{\pi} N^{\frac{3}{2}}}{n+1}\left(\frac{\pi}{n+1}\right)^{2} \exp \left(\frac{-2 N \pi^{2}}{2(n+1)^{2}}\right)(1+o(1)) .
\end{aligned}
$$

Thus,

$$
\frac{\left|D_{2 N, n}\right|}{C_{N}}=\frac{4 \sqrt{\pi} N^{\frac{3}{2}}}{n+1}\left(\frac{\pi}{n+1}\right)^{2} \exp \left(\frac{-N \pi^{2}}{(n+1)^{2}}\right)(1+o(1)) .
$$


Therefore,

$$
\log \left(\frac{\left|D_{2 N, n}\right|}{C_{N}}\right)=\log \left(\frac{4 \pi^{\frac{3}{2}} N^{\frac{3}{2}}}{(n+1)^{3}}\right)-\frac{\pi^{2} N}{(n+1)^{2}}+o(1) .
$$

Multiplying both sides of $(2)$ by $\frac{(n+1)^{2}}{N}$ and letting $N \rightarrow \infty$, we obtain

$$
\lim _{N \rightarrow \infty} \frac{(n+1)^{2}}{N} \log \mathbb{P}_{N}(\max \text { of a Dyck Path with length } 2 N<n)=-\pi^{2} .
$$

Finally, we consider the last possible regime for $n \leq N \ll n^{2}$.

Theorem 3.4. Let $N$ be any positive integer and $x>0$,

- If $n \ll N \ll n^{2}$, then we have

$$
\lim _{N \rightarrow \infty} \frac{N}{2 n^{2}} \log \mathbb{P}_{N}(\text { max height of a Dyck path with length } 2 N>x n) \rightarrow-x^{2} .
$$

- If $n \sim 2 N$, then we have

$$
\begin{aligned}
& \lim _{N \rightarrow \infty} \frac{1}{2 N} \log \mathbb{P}_{N}(\text { max height of a Dyck path with length } 2 N>x n) \rightarrow h(x), \\
& \text { for } 0<x \leq \frac{1}{2}^{-} \text {where } \\
& \qquad h(x)=-\left(x+\frac{1}{2}\right) \log (1+2 x)-\left(\frac{1}{2}-x\right) \log (1-2 x) .
\end{aligned}
$$

Otherwise,

$\lim _{N \rightarrow \infty} \frac{1}{2 N} \log \mathbb{P}_{N}(\max$ height of a Dyck path with length $2 N>x n) \rightarrow-\infty$.

Proof. Let $n \ll N \ll n^{2}$. We start by finding a lower bound for the number of Dyck paths which hit $x n$ at least once in $2 N$ steps. Consider Dyck paths that hit a maximum of $x n$ at the $N t h$ step. We know from [3] that the number of all paths from the origin to the point $(N, x n)$ is given by $\left(\begin{array}{c}N \\ \frac{N+x n}{2}\end{array}\right)$. We 
have to subtract the number of paths which at some point go below the $x$ axis. By the reflection principle this is $\left(\begin{array}{c}N \\ \frac{N+x n}{2}+1\end{array}\right)$. Thus the number of Dyck paths with this property in $N$ steps is

$$
\left(\begin{array}{c}
N \\
\frac{N+x n}{2}
\end{array}\right)-\left(\begin{array}{c}
N \\
\frac{N+x n}{2}+1
\end{array}\right)=\left(\begin{array}{c}
N \\
\frac{N+x n}{2}
\end{array}\right) \frac{x n+1}{\frac{N+x n}{2}+1}
$$

Therefore, the number of Dyck paths in $2 N$ steps with this property is equal to $\left(\begin{array}{c}N \\ \frac{N+x n}{2}\end{array}\right)^{2}\left(\frac{x n+1}{\frac{N+x n}{2}+1}\right)^{2}$. Thus,

$\mathbb{P}_{N}($ max height of a Dyck path with length $2 N>x n)>\frac{\left(\begin{array}{c}N \\ \frac{N+x n}{2}\end{array}\right)^{2}\left(\frac{x n+1}{\frac{N+x n}{2}+1}\right)^{2}}{2^{2 N}}$.

It is easy to show that

$$
\log \left(\begin{array}{c}
N \\
\frac{N+x n}{2}
\end{array}\right)=N \log 2-\frac{(x n)^{2}}{N}+O\left(\frac{1}{N}\right) .
$$

Thus

$\log \left(\begin{array}{c}N \\ \frac{N+x n}{2}\end{array}\right)^{2}\left(\frac{x n+1}{\frac{N+x n}{2}+1}\right)^{2}=\log 2^{2 N}-\frac{2 x^{2} n^{2}}{N}+\log \left(\frac{x n+1}{\frac{N+x n}{2}+1}\right)^{2}+O\left(\frac{1}{N}\right)$.

Therefore,

$\liminf _{N \rightarrow \infty} \frac{N}{2 n^{2}} \log \mathbb{P}_{N}(\max$ height of a Dyck path with length $2 N>x n) \geq-x^{2}$.

To determine an upper bound, we consider all paths that start at the origin, hit $x n$ at least once and can end at any point. Therefore, we need to take $N+x n$ steps out of $2 N$ steps upwards. By the reflection principle the number of such paths is equal to $2\left(\begin{array}{c}2 N \\ N+x n\end{array}\right)$. We thereby obtain,

$\mathbb{P}_{N}($ max height of a Dyck path with length $2 N>x n)<\frac{2\left(\begin{array}{c}2 N \\ N+x n\end{array}\right)}{2^{2 N}}$. 
We can show that

$$
\log \left(\begin{array}{c}
2 N \\
N+x n
\end{array}\right)=\frac{-2 x^{2} n^{2}}{N}+2 N \log (2)+O\left(\frac{1}{N}\right)
$$

Hence,

$\limsup _{N \rightarrow \infty} \frac{N}{2 n^{2}} \log \mathbb{P}_{N}(\max$ height of a Dyck path with length $2 N>x n) \leq-x^{2}$.

Now if we assume $n \sim 2 N$, then if $0<x<\frac{1}{2}$ we deduce

$\mathbb{P}_{N}($ max height of the Dyck path with length $2 N>x 2 N)=\frac{\left(\begin{array}{c}2 N \\ N+x 2 N\end{array}\right)}{2^{2 N}}$,

via same argument. Therefore for $0<x<\frac{1}{2}$, we have

$\log \left(\begin{array}{c}2 N \\ N+x 2 N\end{array}\right)=2 N \log 2-2 N\left(x+\frac{1}{2}\right) \log (1+2 x)-2 N\left(\frac{1}{2}-x\right) \log (1-2 x)$.

So we get

$\lim _{N \rightarrow \infty} \frac{1}{2 N} \log \left(\frac{\left(\begin{array}{c}2 N \\ N+x 2 N\end{array}\right)}{2^{2 N}}\right)=-\left(x+\frac{1}{2}\right) \log (1+2 x)-\left(\frac{1}{2}-x\right) \log (1-2 x)$.

For the case where $x \geq \frac{1}{2}$, we have

$$
\mathbb{P}_{N}(\max \text { height of the Dyck path with length } 2 N=N)=\frac{1}{C_{N}}
$$

since the maximum of a Dyck path cannot be greater than half of the steps and in the case of equality, there is only one path with this property. So

$$
\begin{aligned}
\lim _{N \rightarrow \infty} \frac{1}{2 N} \log \left(\frac{1}{C_{N}}\right) & =\lim _{N \rightarrow \infty} \frac{1}{2 N}\left(\log \left(\frac{1}{4^{N}}\right)+o(1)\right) \\
& =-\log 2 .
\end{aligned}
$$

Note $\lim _{x \rightarrow \frac{1}{2}}\left(-\left(x+\frac{1}{2}\right) \log (1+2 x)-\left(\frac{1}{2}-x\right) \log (1-2 x)\right)=-\log 2$. 
Remark 2. In Theorem 3.4 if we assume $x=1$, we have for $n \ll N \ll n^{2}$

$\lim _{N \rightarrow \infty} \frac{N}{n^{2}} \log \mathbb{P}_{N}(\max$ height of a Dyck path with length $2 N>n) \rightarrow-2$.

Acknowledgments. This paper is supported by University of Ottawa, and NSERC Canada Discovery Grants of Dr. David Handelman. The author wishes to thank Dr. Benoit Collins, for his help, advice and encouragement on this paper. She also acknowledges vital help from Dr. David McDonald for the algebraic proof of Theorem 2.2 .

\section{References}

[1] Chung, K. (1975) Maxima in Brownian excursions. Bull. Amer. Math. Soc. $81,742-745$.

[2] Chung, K. (1976) Excursions in Brownian motion. Ark. Mat. 14, no. 2, 155177. 60J65

[3] Feller, W. (1968) An introduction to probability theory and its applications. Vol. I. Third edition John Wiley and Sons, Inc. York-LondonSydney.

[4] Flajolet, P.; Sedgewick, R. (2009) Analytic combinatorics Cambridge University Press, Cambridge.

[5] Kaigh, W. D. (1976) An invariance principle for random walk conditioned by a late return to zero. Ann. Probability 4, no. 1, 115-121.

[6] Kennedy, Douglas P. (1976) The distribution of the maximum Brownian excursion. J. Appl. Probability 13 , no. 2, 371-376.

[7] Khorunzhiy, O; Marckert, J.F. (2009) Uniform bounds for exponential moments of maximum of Dyck paths. Elect. Comm. in Probab. 14, 327333.

[8] Hiai, F; Petz, D. The semicircle law, free random variables and entropy. (English summary) Mathematical Surveys and Monographs, 77. American Mathematical Society, Providence, RI.

[9] Rouault, Alain. Private communication. 